\title{
Transmission Properties of the Composite Structure Constructed by Air and Single Negative Meta- materials
}

\author{
T.H. Feng \\ College of Electrical and Information Engineering \\ Xuchang University \\ Xuchang, China
}

\begin{abstract}
In order to explore the applications of single negative meta-materials (SNM) in wireless system, the electromagnetic properties of composite structure constructed by the SNM and air are investigated by means of transfer-matrix method. The results show that the tunneling phenomenon will happen in the sandwich structure composed of epsilon-negative meta-materials (ENM), mu-negative meta-materials (MNM) and air under proper parameters. In addition, the electric and magnetic fields are localized at different interface respectively and the electric field is localized at the interface of MNM and air but the magnetic field is at the interface of ENM and air. With these features, the present structure may have important potential applications in wireless transmission.
\end{abstract}

Keywords-metamaterial; single negative; wireless transmission

\section{INTRODUCTION}

As we know, the traditional wireless system based on antennas has many disadvantages. First, the antenna is omnidirectional and the receiver just utilizes a small part of the emissive energy. Moroever, the antenna generally has to work endlessly since it can not know whether the receivers are running. A mass of energies are wasted for these tow aspects. In additon, one generally magnify the emanant power to enhance the received singnal intensity. However, the arisen problem is that the enhanced signals will be bad for people's health. For solving these issues, new methods must be explored.

$\mathrm{s}$ in wireless transmission.

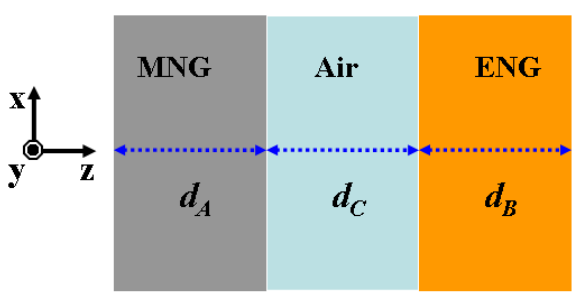

FIGURE I. SCHEMATIC OF THE COMPOSITE STRUCTURE COMPOSED OF MNM, AIR AND ENM

\section{TRANSMISSION PROPERTIES OF MNM-AIR-ENM STRUCTURE}

Fig. 1 is the schematic of our structure where the ENMMNM heterostructure is inserted by air and the ENM and
In the last few years, single negative meta-materials (SNM) including epsilon-negative meta-materials $\left(\varepsilon<0,{ }^{\mu}>0\right)$ and mu-negative meta-materials ( $\mu<0, \varepsilon>0$ ), have drawn many people's interests for their extraordinary properties [1-8]. For sole epsilon-negative meta-materials (ENM) or mu-negative meta-materials (MNM), they just support decaying waves and are opaque since their wavevectors are complex. However, some composite structures based on SNM can be transparent to electromagnetic (EM) waves and have many novel properties and important applications [1-7]. For instance, the waves can tunnel through a heterostructure constructured by ENM and MNM and the image displacement and image reconstruction can be realized by the SNM heterostructure. [1-3]. The selfcollimation of a relatively broadband light beam is realized for a long distance in the photonic crystals made of ENM and MNM, and both far and near fields can be collimated and boosted greatly, which will play an important role in the applications involving slow wave or nonlinear effect [7]. In this paper, The EM properties of the sandwich structure made of ENM, MNM and air are investigated numerically. The results demonstrate that the tunneling phenomenon will happen in the sandwich structure composed of ENM, MNM and air under proper parameters. In addition, the electric and magnetic fields are localized at different interface respectively and the electric field is localized at the interface of MNM and air but the magnetic field is at the interface of ENM and air. The present structure may have potential application

MNM are separated. For this MNM-Air-ENM sandwich structure, does the tunneling phenomenon happen? Now, the EM properties of this structure will be studied numerically. We suppose that

$$
\varepsilon_{\mathrm{MNM}}=\varepsilon_{\mathrm{A}}, \mu_{\mathrm{MNM}}=\mu_{\mathrm{A}}-\frac{\alpha}{\omega^{2}}
$$

In MNM and

$$
\varepsilon_{\mathrm{ENM}}=\varepsilon_{\mathrm{B}}-\frac{\beta}{\omega^{2}}, \mu_{\mathrm{ENM}}=\mu_{\mathrm{B}}
$$

In ENM, where $\omega$ is the frequency in unit of $\mathrm{GHz}$ [8]. It is assumed that $\varepsilon_{\mathrm{A}}=\mu_{\mathrm{B}}=1, \mu_{\mathrm{A}}=\varepsilon_{\mathrm{B}}=1$, and $\alpha=\beta=400$. The thicknesses of MNM and ENM layers are chosen to be $\mathrm{dA}=20$ 
$\mathrm{mm}$ and $\mathrm{dB}=20 \mathrm{~mm}$ respectively and the air layer is $\mathrm{dC}=20$ $\mathrm{mm}$. Assuming that a transverse electric wave is normally incident onto the MNM-Air-ENM structure surrounded by air, the transmission properties

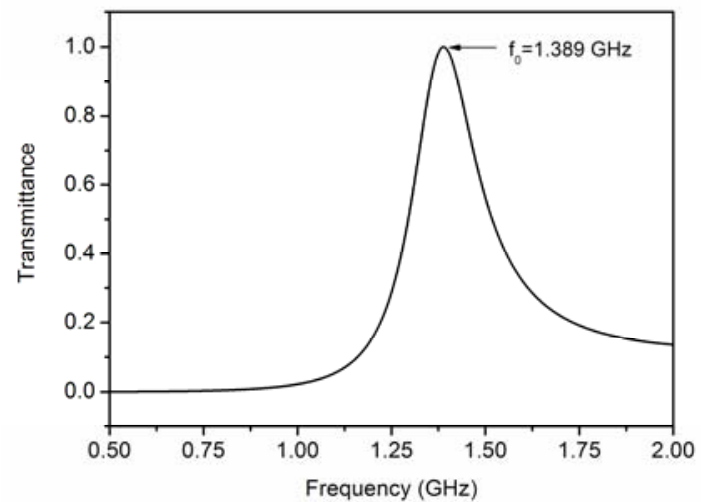

(a)

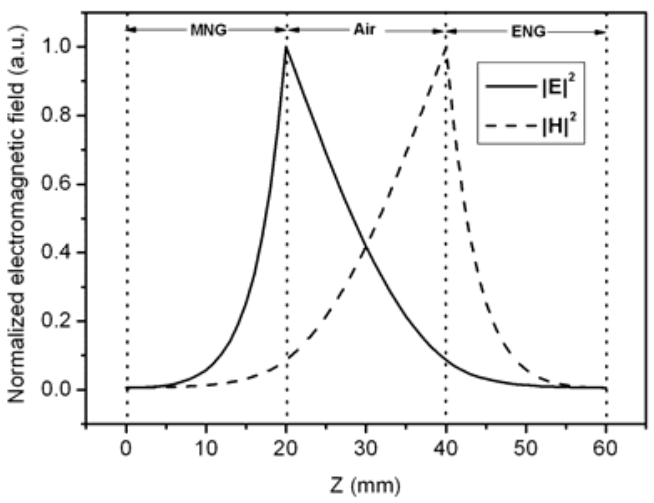

(b)

FIGURE II. (a) THE TRANSMISION CURVES OF THE MNM-AIR-ENM STRUCTURE WITH THE THICKNESSES OF DA $=\mathrm{DB}=\mathrm{DC}=20 \mathrm{~mm}$. (b) THE EM FIELD DISTRIBUTION IN THE MNM-AIR-ENM STRUCTURE

Of MNM-Air-ENM structure can be calculated by transfermatrix method. We give the transmission curve of the MNMAir-ENM structure in Fig. 2(a). Obviously, the tunneling phenomenon happens and the MNM-Air-ENM structure is completely trasparant to the EM waves at $\mathrm{f0}=1.389 \mathrm{GHz}$. This means that a new wireless transmission system can be designed utilizing the present MNM-Air-ENM composite structure. In addition, we also investigated the field localization in the MNM-Air-ENM structure and Fig. 2(b) illustrates the electrmagnetic field distribution in the composite structure at the frequency of $1.389 \mathrm{GHz}$. It is interesting that the magnetic and electric field is localized at different position. At the interface of MNM and air layers the magnetic field is localized and the localizing electric field lies surprisingly between ENM and air layers. Considering that the localized magnetic field generally do not interact with surrounding objects, it will be good for the people's health when the ENM is employed to act as a receiver.

To investigate the effect of thickness of air on the transmission properties of the MNM-Air-ENM sandwich structure, we also calculated the transmission curves of the composite structure under the different thickness of air, as illustrated in Fig. 3(a). Thereinto, the thickness of ENM and MNM layers is invariable and they are also $\mathrm{dA}=\mathrm{dB}=20 \mathrm{~mm}$. In Fig. 3(a), it is clear that the

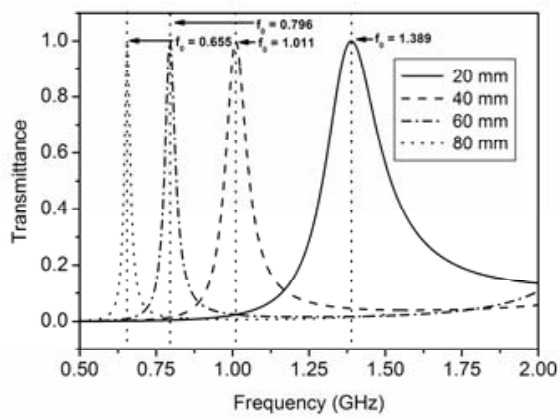

(a)

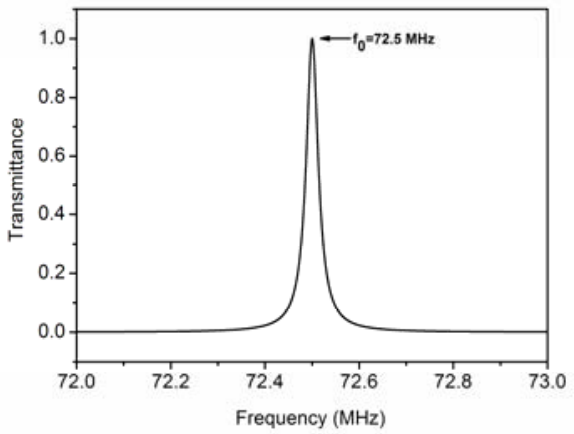

(b)

FIGURE III (a) THE TRANSMISSION CURVES OF THE MNMAIR-ENM STRUCTURE WITH DIFFERENT THICKNESS OF AIR. (b) THE TRANSMITTANCE OF THE MNM-AIR-ENM STRUCTURE WITH THE THICKNESSES OF $d_{A}=20 \mathrm{~mm}, d_{C}=1000 \mathrm{~mm}$ AND $d_{B}=20 \mathrm{~mm}$.

Tunnelling frequency shifts gradually to lower frequencies with the increase of air thickness. Furthermore, for the sake of exploring whther the tunnelling phenomenon still occurs when the thickness of air is enough large compared with SNG layers, we also calculated the transmittance of the MNM-Air-ENM structure under the air thickness of $\mathrm{dC}=1000 \mathrm{~mm}$. Hereinto, the thickness of the MNM and ENM layers are also $\mathrm{dA}=\mathrm{dB}=$ $20 \mathrm{~mm}$. In this case, the air is 50 times the thichness of the SNM layers. The transmission curve is depicted in Fig. 3(b) and obviously, the EM waves still tunnel through the composite structure at the frequency f0 $=72.5 \mathrm{MHz}$. Therefore, according to the definition of mid-range in [9], a highly efficient wireless device with its functional region beyond mid-range can be obtained by using our structure.

\section{TRANSMISSION PROPERTIES OF (MNM-AIR-ENM)8 STRUCTURE}

In additon, we also studied the transmitting propetries of periodic structure of (MNM-Air-ENM)8 (here, 8 denotes the periodic number), aiming to realizing long distance transmission by transfer station. Fig. 4(a) gives the comparison of the transmittance curve of the structure MNM-Air-ENM and periodic structure (MNM-Air-ENM)8 under the parameters of $\mathrm{dA}=\mathrm{dB}=\mathrm{dC}=20 \mathrm{~mm}$, and Fig. 4(b) 


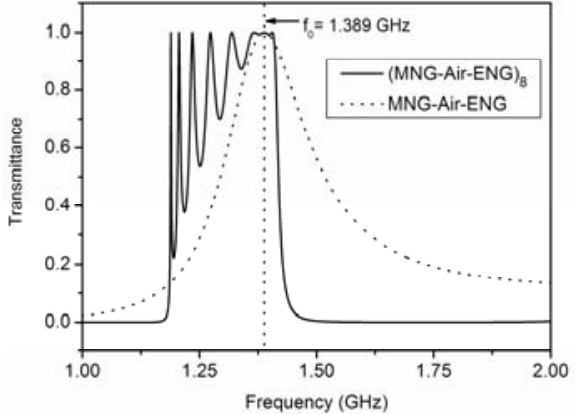

(a)

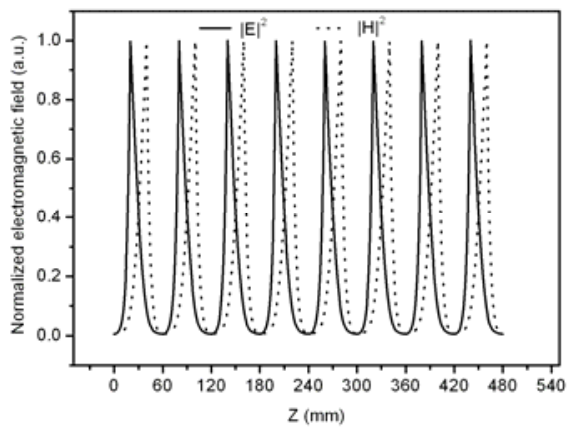

(b)

FIGURE IV.

(a) COMPARISON OF TRANSMISSION CURVES OF THE STRUCTURE MNM-AIR-ENM AND THE PERIODIC STRUCTURE (MNM-AIR-ENM)8. (b) THE EM FIELD DISTRIBUTION IN THE PERIODIC STRUCTURE (MNM-AIR-ENM)8.

Illustrates the EM field distribution in the periodic structure (MNM-Air-ENM)8 for the frequency f0 $=1.389 \mathrm{GHz}$. Obviously, the periodic structure (MNM-Air-ENM)8 still be transparent to EM waves at the quondam tunneling frequency $\mathrm{f0}=1.389 \mathrm{GHz}$ and the magnetic and electric field is still separated and localized at different interface. Therefore, utilizing the periodic structure (MNM-Air-ENM)n, long distance transmission based on transfer station can be realized.

\section{CONCLUSION}

In conclusion, we investigated theoretically the transmitting properties of MNM-Air-ENM and (MNM-Air-ENM)8 structure. The results demonstrate that the EM waves can trransmit through the MNM-Air-ENM and (MNM-Air-ENM)8 structure under proper condition. Moreover, the EM field distribution is interesting and the magnetic and electric field is localized at the interface of ENM-Air and MNM-Air respectively. Therefore, the composite structure constructed by air and single negative meta-materials may may have important applications in wireless transmission system with the advantages of high efficiency and health.

\section{ACKNOWLEDGMENTS}

Support from the Key Research Project for Science \& Technology in the Education Department of Henan Province (grant 14B140011) and the Natural Science Foundation of Xuchang University (grant 2015105).

\section{REFERENCES}

[1] Andrea Alù, and Nader Engheta, "Pairing an Epsilon-Negative Slab With a Mu-Negative Slab: Resonance, Tunneling and Transparency," IEEE Transactions on Antennas and Propagation, vol. 51, pp. 2558-2571, 2003.

[2] Timothy Fujishige, Christophe Caloz, Tatsuo Itoh, "Experiment demonstration of transparency in the ENG-MNG pair in a CRLH transmission-line implementation,” Microwave and Optical Technology Letters, vol. 46, pp. 476-481, 2005.

[3] Y.F. Shen, C. Xu, Y.F. Tang, et al., "Ultra-compact subwavelENMth and single-mode cavity resonator," Chin. Phys. Lett., vol. 23, pp. 16001602, 2006.

[4] G.S. Guan, H.T. Jiang, H.Q. Li, et al., "Tunneling modes of photonic heterostructures consisting of single-negative materials,” Applied Physics Letters, vol. 88, pp. 211112, 2006.

[5] T. Feng, L. Fan Y.H. li, et al., "Experiment study of tunneling phenomenon occurring in the photonic heterostructure consisting of negative materials,” The European Physical Journal B, vol. 69, pp. 315319, 2009.

[6] T.H. Feng, Y.H. Li, J.Y. Guo, et al., "Highly localized mode in a structure made of epsilon-negative and mu-negative metamaterial," Jounal of Applied Physics, vol. 104, pp. 013107, 2008.

[7] Z.L. Wang, H.T. Jiang, Y.H. Li, H. Chen, "Enhancement of selfcollimated fields in photonic crystals consisting of two kinds of singlenegative materials,” Optics Express, Vol. 18, pp. 14311-14318, 2010.

[8] R. Zeng, Y.P. Yang, S.Y. Zhu, "Casimir force between anisotropic single-negative meta-materials," Physical Review A, vol. 87, pp. 063823, 2013.

[9] Andre Kurs, Aristeidis Karalis, Robert, et al., "Wireless power transfer via strongly coupled magnetic resonances,” Science, vol. 317, pp. 83-86, 2007. 